\title{
Spectral gain investigation of large size OPCPA based on partially deuterated KDP
}

\author{
Marco Galimberti*, Alexis Boyle, Ian O. Musgrave, Pedro Oliveira, Dave Pepler, Waseem Shaikh, Trevor B. Winstone, \\ Adam Wyatt, Cristina Hernandez-Gomez
}

Central Laser Facility, STFC Rutherford Appleton Laboratory, Chilton, Didcot, OX11 OQX, UK

\begin{abstract}
The Optical Parametric Chirped Pulse Amplification is one of the most promising techniques to deliver 20PW laser system. The already available KD*P in large size is a good candidate as nonlinear crystal. In this article we report the experimental analysis of the spectral small signal gain for $\mathrm{KD}^{*} \mathrm{P}$ at $70 \%$ deuteration level for different phase matching and non-collinear angle. The data is also compared with a theoretical model.
\end{abstract}

\section{Introduction}

The Central Laser Facility has made leading contributions to the development of Optical Parametric Chirped Pulse Amplification. Through a series of projects the technique has been developed from preamplifiers to feasibility tests at large aperture. The culmination of this work is the proposal for and development of a 20PW upgrade to the current Vulcan laser facility [1]. The new beam line will be fully integrated with the existing $1 \mathrm{PW}$ beam line enabling new types of experiments $1 \mathrm{PW}+20 \mathrm{PW}$. In addition to the peak power the requirement on the laser pulse will be high contrast, good focusability and able to reach intensities greater than $10^{23} \mathrm{~W} \mathrm{~cm}^{-2}$.

Whilst funding is being sought to fully deliver this goal a technology development programme for multi-PW OPCPA systems has been established to ensure that the relevant components required are available when funding is identified.

Key components in the OPCPA are the nonlinear crystals, responsible to the amplifications. To de-risk the project, but also to improve the knowledge and investigate ways to increase the project specifications, a dedicated laboratory was setup to investigate large size OPCPA system.

The deuteration level of $\mathrm{KD}^{*} \mathrm{P}$ has an impact in the absorption in the NIR: while the signal is not impacted from it, the idler could be affected reducing the gain and the efficiency of the amplification. Different deuteration change also the phase matching of the process, having an impact on the amplification bandwidth achievable.

While there is a great variety of nonlinear crystals, only a small number are available in large size $(>100 \mathrm{~mm})$. One of the most used crystal already available in large size is the $\mathrm{KD} * \mathrm{P}$, used in large fusion laser system, like $\mathrm{NIF}$. KD*P is similar to the KDP, where the hydrogen has been partially substituted by deuterium. The degree

Corresponding author: marco.galimberti@stfc.ac.uk of substitution is called deuteration level and it has influence on the optical properties of the crystal, mainly on the refractive index and the absorption in the near infrared.

A detailed theoretical and numerical study on the influence of the deuteration level on the spectral gain of an OPCPA is available in literature [2], where an optimal spectral gain was obtained for different deuteration level, by changing the phase matching angle and the pump non-collinear angle. The result are shown in fig. 1 .

From this study, partially deuterated $\mathrm{KD} * \mathrm{P}$ could be used in the 20PW project. While the results are promising, an experimental study of the spectral gain of an OPCPA is required to validate the theoretical model.

\section{Experimental Investigation}

Based on the 20PW project [1], the high energy OPCPA stages required around $600 \mathrm{~J}$ of pump laser and large beam size $(20 \mathrm{~cm})$, not easy available. Instead of the final

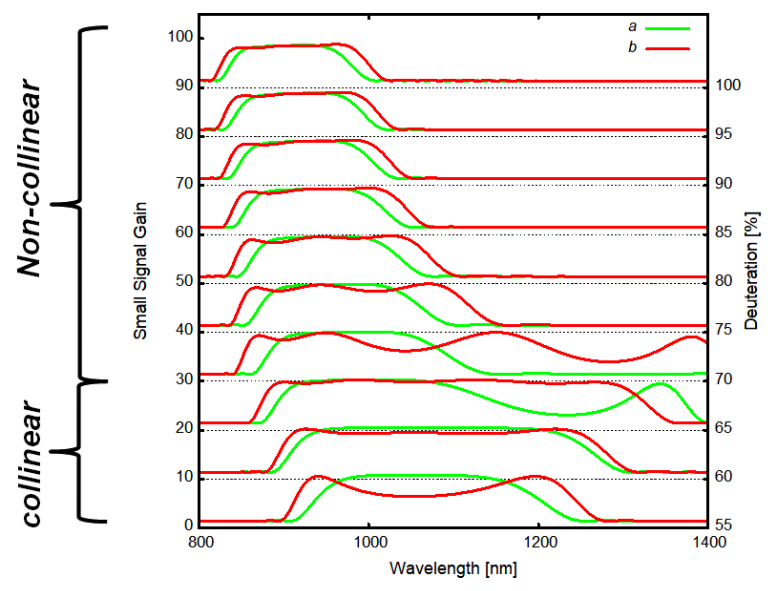

Fig. 1. Optimal spectral small signal gain for $\mathrm{KD} * \mathrm{P}$ with different deuteration level [2]. 


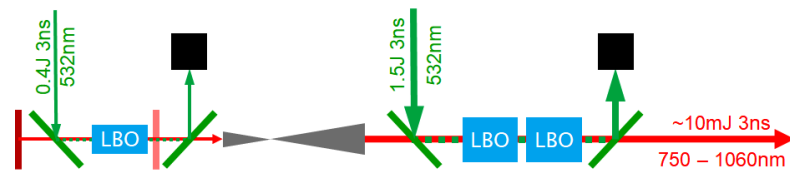

Fig. 2. Optical layout of the OPG source

amplifier, the study was based on the booster stage, where the pump beam size is $35 \mathrm{~mm}$ with energies of $15 \mathrm{~J}$ at $526.5 \mathrm{~nm}$. The main required to perform the study are:

- Crystal samples;

- Broad band signal, to be used as a seed;

- Pump laser and OPCPA setup;

- Procedure to find the phase matching.

Different deuterated $\mathrm{KD}^{*} \mathrm{P}$ were obtained. For this article we chose the $70 \%$ deuterated $\mathrm{KD}^{*} \mathrm{P}$, more readily available in large size. The test crystal was $50 \mathrm{~mm} \mathrm{x}$ $50 \mathrm{~mm}, 64 \mathrm{~mm}$ long.

\subsection{Signal generation}

The required seed need to cover a large spectral range, from $750 \mathrm{~nm}$ to $1050 \mathrm{~nm}$, comparable pulse duration and synchronizable with the pump laser and of energies of the order of few mJ.

Different techniques could be used to generate this seed. Typically fs laser system are used, followed by OPG or white light generation. Unfortunately, those systems are complex and require a stretcher to match the pulse length to the pump laser.

In our case we opted for a simpler approach to directly generate a seed pulse with a suitable pulse duration and energy, based on optical parametric oscillator and OPA. A schematic of our scheme is shown in fig. 2. The seed generation was performed by a resonant cavity around a $38 \mathrm{~mm}$ long Type I LBO crystal, pumped by a $0.4 \mathrm{~J} 3 \mathrm{~ns}$ at $532 \mathrm{~nm}$ providing with high single pass gain in excess of 10 . The cavity length allowed 3 round trips during the pump pulse duration, generating laser pulses exceeding $30 \mathrm{~mJ}$. The central wavelength could be tuned by varying the phase matching angle of the crystal [3].

The generated beam was spatially filter and expanded to $10 \mathrm{~mm}$ before be amplified by an additional dual stage OPA. After the spatial filter the spectrum was maintained but the energy was reduced to $10 \mathrm{~mJ}$.

The second OPA amplification stage was constituted by two $13 \mathrm{~mm}$ thick LBO crystal pumped by $1.5 \mathrm{~J}$ at a fluence of $1.9 \mathrm{~J} / \mathrm{cm}^{2}$. At the output a pulse energy of 90 $\mathrm{mJ}$ was obtained, with a stability of $6 \% \mathrm{rms}$. By changing the phase matching angles, a tuneable range of $300 \mathrm{~nm}$ from 750 to $1050 \mathrm{~nm}$ was achieved as shown in fig. 3 .

\subsection{Pump laser and OPCPA setup}

To deliver a pulse energy of the order of $15 \mathrm{~J}$ at $526.5 \mathrm{~nm}$ in $3 \mathrm{~ns}$ needed for the test, a Nd:Glass laser system was setup, basing the design on the rod chain of the Vulcan laser system. Due to the properties of the OPCPA,

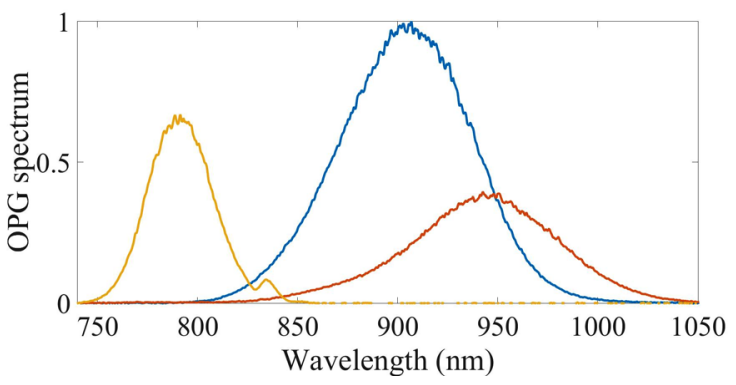

Fig. 3. OPG spectra, centred at 790,910 and $950 \mathrm{~nm}$ obtained by changing the phase matching angle.

temporal and spatial properties of the laser pulse are crucial to achieve the required amplification. More in detail, a flat top uniform beam profile, single frequency (single longitudinal mode) and temporal square pulses is needed. A layout of the pump laser is shown in fig. 4 [3, 4].

The laser pulse was generated by a long pulse front end with a programmable temporal pulse shape, capable to deliver around $1 \mathrm{~mJ}$ on a single frequency at $1053 \mathrm{~nm}$.

The pulse was spatially filtered by an diffraction limited pinhole and amplified by a series of flash pumped phosphate Nd:Glass amplifiers, with the final amplifier was based on a $45 \mathrm{~mm}$ rod in a double pass configuration. The system was capable to deliver reliable pulses of $30 \mathrm{~J}$ at $1053 \mathrm{~nm}$ with pulse length of $3 \mathrm{~ns}$.

The infrared pulse was frequency doubled by a type I KDP $22 \mathrm{~mm}$ long. The resulting green pulses were sent to the OPCPA crystal by a vacuum telescope, with energy of $15 \mathrm{~J}$.

The signal, generated by the OPG, was expanded to $35 \mathrm{~mm}$ by an achromatic vacuum telescope and sent to the OPCPA crystal. A full set of diagnostics, including $\mathrm{NF}$, FF and spectrum, were setup to investigate the amplification process.

Because the repetition rate of the pump laser was low, with a shot every 5 minutes, a procedure to tune the phase matching of the OPCPA crystal was necessary. To investigate different phase matching angle in one shot, using the spatial extension of the beam, a cylindrical negative lens was inserted just before of the OPCPA crystal. In this way, the divergence of the pump and the signal beams was resulting in different incidence angles

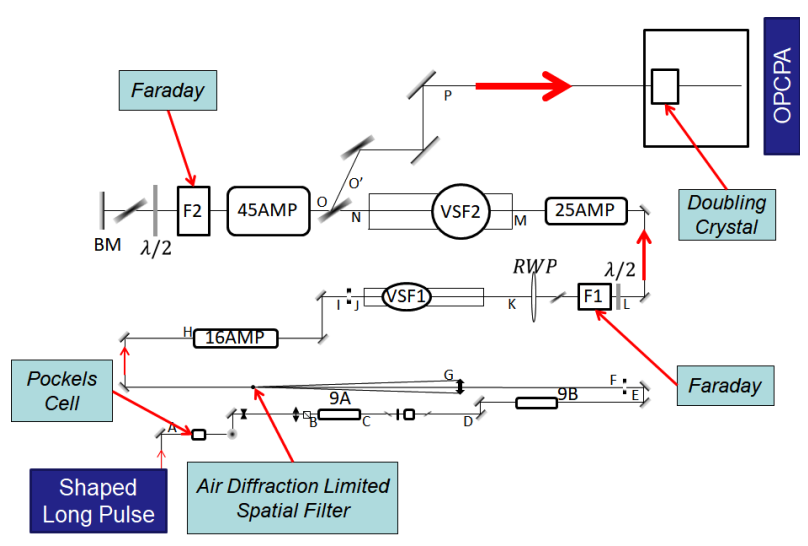

Fig. 4. Layout of the pump laser. 


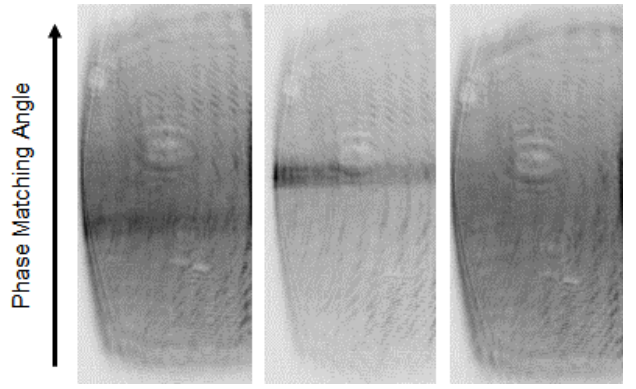

Fig. 5. Near field images during phase matching tuning. The three images were acquired at different phase matching angles.

on the crystal along one spatial direction. In case of phase matching, a line in the near field image, related to the region with gain, should appear.

A set experimental images is shown in fig. 5. The line where there was gain is clearly visible in the first and second image. Each image are related to different angular position of the crystal, and it clearly visible the line moving with the crystal.

\section{Results and discussion}

A complete phase matching scan was performed for four non-collinear angles of the pump. Due to the characteristics of the seed, for a single position of phase marching and non-collinear angle various shots were taken, to cover all the required spectral region. The full set of data was acquired over three month with hundreds of shots.

The data was analysed numerically to join the different acquisition and to extract the spectral gain. The results are shown in fig. 6 . The phase matching angle $\theta$ is experimental and it is subjected of a systematic error of

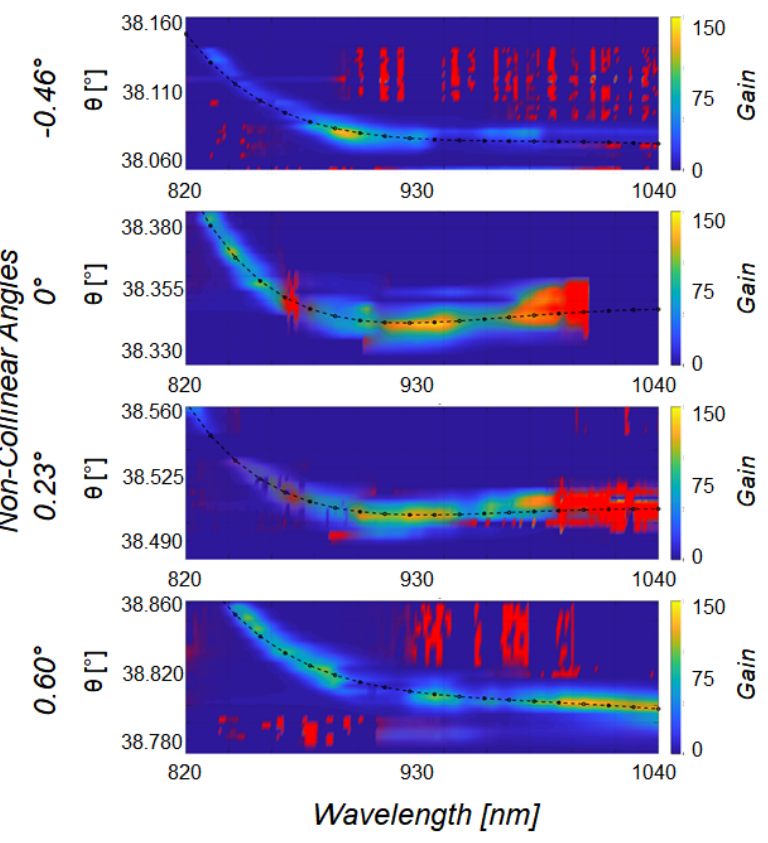

Fig. 6. Measured spectral small signal gain for different noncollinear angles and phase matching angles $\theta$.

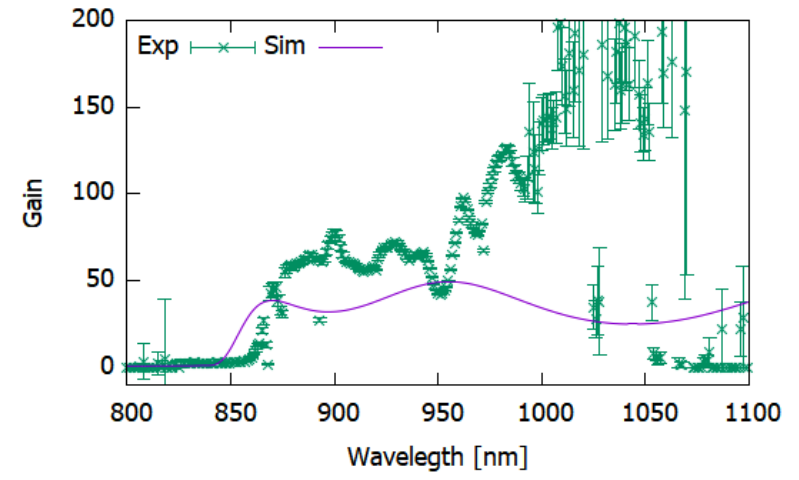

Fig. 7. Comparison between experimental and simulated small signal gain.

about $0.5^{\circ}$, due to the precision of the cut of the crystal. The measured gain was approximately in agreement with the theoretical model.

Selecting the data with maximum spectral bandwidth for the non-collinear angle $0.23^{\circ}$, the data could be more easily compared with the theoretical model. Best match was found for a phase matching angle of $38.32^{\circ}$. From the comparison (fig. 7), theoretical model predicted gain at lower wavelength than the experimental data.

Reconsidering the entire dataset, a possible explanation of this mismatch was found analysing the phase matching at different deuteration level. As shown in fig. 6 , the data was in agreement with the phase matching curve, shown in dashed line, for deuteration level of $66 \%$.

\section{Conclusion}

The small signal gain of an OPCPA based on partially deuterated $\mathrm{KD} * \mathrm{P}$ at $70 \%$ was investigate. To perform the study a broadband tuneable source was setup within a $15 \mathrm{~J}$ pump laser and a laboratory.

A complete dataset of phase matching and non-collinear angles was acquired. A preliminary analysis showed a good agreement between the experimental data and the theoretical model if a slightly different deuteration level is considered $(66 \%)$.

Future work will be focused on investigating different deuteration level and to improve the data analysis.

\section{Bibliography}

1. C. Hernandez-Gomez et al, 'The Vulcan 10 PW Project: Facility Design Report', Technical Report, Central Laser Facility, STFC, 2010.

2. M. Galimberti et al, Optics Coms. 309, 80-84 (2013)

3. I. Musgrave et al, Proceedings of ICUIL conference 2014, GOA, India

4. M. Galimberti et al. The Review of Laser Engineering: vol. 42(2), 137-140 (2014) 